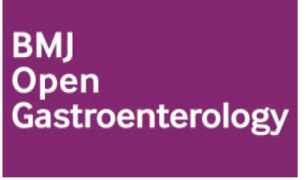

\title{
Developing patient-orientated Barrett's oesophagus services: the role of dedicated services
}

\author{
Elizabeth Ratcliffe (D) ,1,2 James Britton, ${ }^{3}$ Shaheen Hamdy, ${ }^{2,3}$ \\ John McLaughlin (iD) ,2,3 Yeng Ang ${ }^{2,3}$
}

To cite: Ratcliffe E, Britton J, Hamdy S, et al. Developing patient-orientated Barrett's oesophagus services: the role of dedicated services. BMJ Open Gastro 2022;9:e000829. doi:10.1136/ bmjgast-2021-000829

Received 11 November 2021 Accepted 9 January 2022
Check for updates

(C) Author(s) (or their employer(s)) 2022. Re-use permitted under CC BY-NC. No commercial re-use. See rights and permissions. Published by BMJ.

${ }^{1}$ Gastroenterology, Wrightington Wigan and Leigh NHS

Foundation Trust, Leigh, UK

${ }^{2}$ School of Medical Sciences, The University of Manchester Faculty of Biology Medicine and Health, Manchester, UK

${ }^{3}$ Department of

Gastroenterology, Northern Care Alliance NHS Foundation Trust, Salford, UK

Correspondence to Dr Elizabeth Ratcliffe; elizabeth.ratcliffe@wwl.nhs.uk

\section{ABSTRACT}

Introduction Barrett's oesophagus (B0) is common and is a precursor to oesophageal adenocarcinoma with a $0.33 \%$ per annum risk of progression. Surveillance and follow-up services for B0 have been shown to be lacking, with studies showing inadequate adherence to guidelines and patients reporting a need for greater disease-specific knowledge. This review explores the emerging role of dedicated services for patients with B0.

Methods A literature search of PubMed, MEDLINE, Embase, Emcare, HMIC, BNI, CiNAHL, AMED and PsycINFO in regard to dedicated $\mathrm{BO}$ care pathways was undertaken. Results Prospective multicentre and randomised trials were lacking. Published cohort data are encouraging with improvements in guideline adherence with dedicated services, with one published study showing significant improvements in dysplasia detection rates. Accuracy of allocation to surveillance endoscopy has been shown to hold cost savings, and a study of a dedicated clinic showed increased discharges from unnecessary surveillance. Training modalities for B0 surveillance and dysplasia detection exist, which could be used to educate a BO workforce. Qualitative and quantitative studies have shown patients report high levels of cancer worry and poor disease-specific knowledge, but few studies have explored follow-up care models despite being a patient and clinician priority for research.

Conclusions Cost-benefit analysis for dedicated services, considering both financial and environmental impacts, and more robust clinical data must be obtained to support this model of care in the wider health service. Greater understanding is needed of the root causes for poor guideline adherence, and disease-specific models of care should be designed around clinical and patientreported outcomes to address the unmet needs of patients with $\mathrm{BO}$.

\section{INTRODUCTION}

Barrett's oesophagus (BO) is a precursor to oesophageal adenocarcinoma (OAC) with a rate of progression from non-dysplastic Barrett's oesophagus (NDBO) to cancer of around $0.33 \%$ per annum. ${ }^{12}$ Rates of $\mathrm{BO}$ are rising in more socioeconomically high-income countries even accounting for
Summary box

What is known

- Dedicated services are a key research priority for patients and clinicians.

- Current practice shows poor adherence to biopsy protocols with significant levels of missed dysplasia.

What this literature review shows

- There are few studies published; one study to date has shown significantly improved dysplasia detection rates.

- One systematic review showed improvement with dedicated services but was based on only a few studies.

- Data presented at conferences from units in the UK have shown improved adherence to biopsy protocols and documentation without clarity on transformation impact.

What needs to be done

- Large multicentre studies to determine the benefit of dedicated services and look for improved outcomes for patients in real terms: dysplasia detection, oesophageal adenocarcinoma detection and survival.

- Cost-benefit analysis should also be performed to show value (eg, quality-adjusted life years).

- More should be done to improve training and quality of Barrett's oesophagus (B0) surveillance.

- More must be done to look at BO follow-up care pathways and patient education from the patient perspective and to use patient-reported outcomes to shape practice.

- Surveillance has a potentially high environmenta impact; work is needed to model the sustainability and carbon impact of different pathways, and whether dedicated services and non-endoscopic sampling methods may help streamline care to those who most need it.

advances in endoscopy practice. ${ }^{3}$ Cancer Research UK states that $59 \%$ of all cases of oesophageal cancer are preventable and that it carries a dismal $12 \%$ 10-year survival. ${ }^{4}$

Once BO is diagnosed, patients are offered surveillance endoscopy every $2-5$ years, depending on the length of their $\mathrm{BO}$, which 


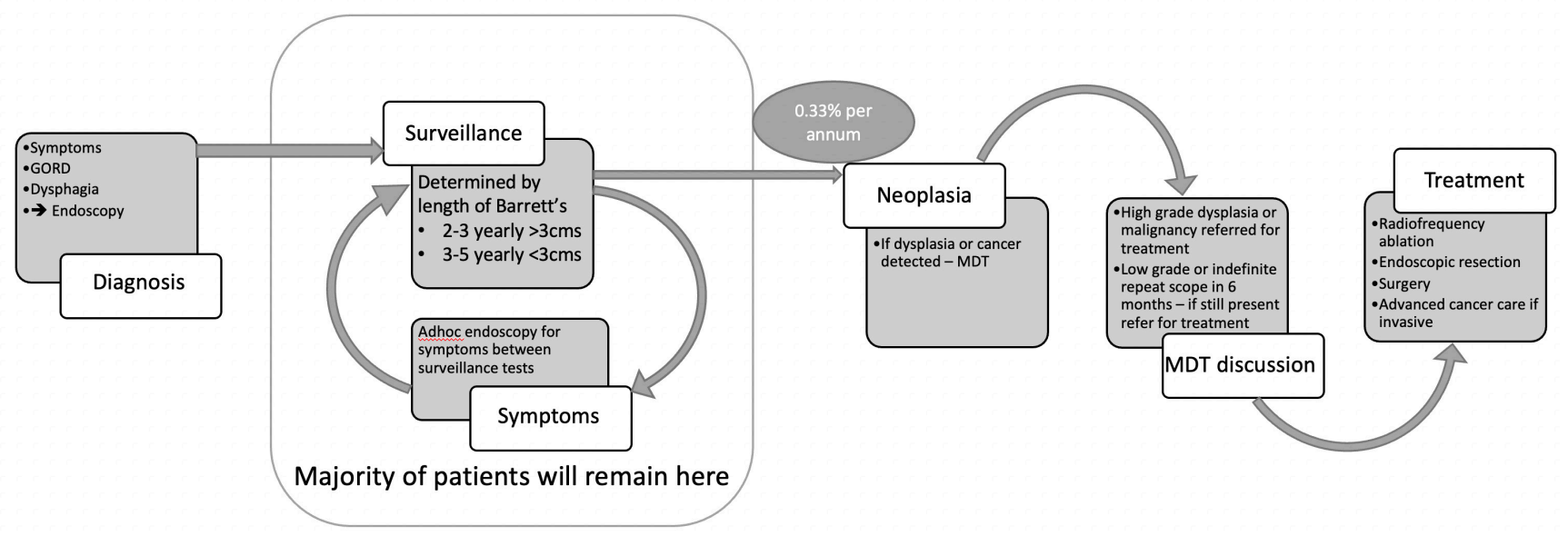

Figure 1 Schematic of a patient journey with Barrett's oesophagus. The majority of patients will remain in the surveillance programme, presenting with symptoms ad hoc as required. Of these patients, $0.33 \%$ per annum may progress to malignancy and dysplasia (Hvid-Jenson et al ${ }^{1}$ ). GORD, gastro-oesophageal reflux disease; MDT, multidisciplinary meeting.

is predominantly performed on routine upper gastrointestinal (UGI) endoscopy lists (figure 1 ). ${ }^{5}$ Standard reporting of results is expected as per the British Society of Gastroenterology (BSG) guidelines ${ }^{5}$ and Seattle protocol biopsies (quadrantic samples at every $2 \mathrm{~cm}$ of the $\mathrm{BO}$ segment) are taken along with targeted samples of lesions. ${ }^{6}$ All patients newly diagnosed with BO should be seen in an outpatient clinic to discuss their diagnosis according to the BSG guidelines. ${ }^{5}$ Studies have shown that patients lack disease-specific knowledge and overestimate their cancer risk with corresponding cancer worry. ${ }^{7-9}$ Retrospective studies suggest endoscopic surveillance of patients with BO improves outcomes, ${ }^{10}{ }^{11}$ and adherence to Seattle protocol biopsies has been shown to be important for the detection of dysplasia. ${ }^{12}$ However, BO surveillance endoscopy has often been shown to be performed inadequately, ${ }^{13}$ with longer segments routinely underbiopsied. ${ }^{14}$ Missed dysplasia is common; a meta-analysis showed $25 \%$ of OAC is missed at an index BO endoscopy, ${ }^{15}$ and a UK-based study suggested $12.7 \%$ was missed $>3$ months after diagnosis of $\mathrm{BO}{ }^{16}$

Patients can also be left on routine surveillance inappropriately or surveyed too frequently ${ }^{17}$; overdiagnosis of $\mathrm{BO}$ at the gastro-oesophageal junction is frequent; and the majority of cases with $\mathrm{BO}$ will die of another disease than OAC. ${ }^{18}$ Information and decision making around multicomorbid patients has been neglected in studies, but guidelines advise clinicians to consider this in decisions around initiating and stopping surveillance. ${ }^{5} 19$ Individual risk stratification methods to aid surveillance decisions are being investigated, ${ }^{20-22}$ and non-endoscopic oesophageal sampling methods have been devised, which may support streamlining of endoscopy use.

Dedicated BO services as a model of care are recognised in the tertiary setting, with advanced endoscopy for BO lesions centralised in referral centres. However, most initial diagnosis of $\mathrm{BO}$, surveillance and follow-up of
NDBO occur in the general setting. A research priority setting exercise published in 2017 was undertaken to determine the top 10 research priorities for $\mathrm{BO}^{23}$; the fourth priority was 'Should surveillance and new patient clinics for BO be done by a dedicated service? How would this compare with existing standards of practice in the UK, and what effect would this have on patients (eg, precancer diagnosis rates, patient education, quality of life and satisfaction)?'

This review explores how well this priority is covered in the literature at present and the areas of unmet need in BO that a dedicated service could address.

\section{METHODS}

Using National Institute of Healthcare Excellence Healthcare Databases Advance Search, we performed a literature search of PubMed, MEDLINE, Embase, Emcare, HMIC, BNI, CiNAHL, AMED and PsycINFO to September 2020 was performed. A separate search was performed of the Cochrane database and review articles, and key papers' reference lists were reviewed for further relevant papers. Search strategy was undertaken as follows: ("dedicated" AND "Barrett's Oesophagus"), (("dedicated" AND "Barretts oesophagus") AND ("clinic" OR "endoscopy")), ("Barretts oesophagus" AND ("clinic" OR "endoscopy")), (("dedicated service" AND "gastroenterology") AND (Endoscopy OR clinic)), (dedicated endoscopy for Barrett's oesophagus), (dedicated clinic for Barrett's oesophagus).

Reinforcing the novel nature of dedicated services for NDBO, we found that there were few fully published studies with no randomised controlled trials and only a few that were multicentre.

\section{Dedicated BO surveillance services}

Studies comparing types of Barrett's services are summarised in table 1 . Only one study published in the UK 


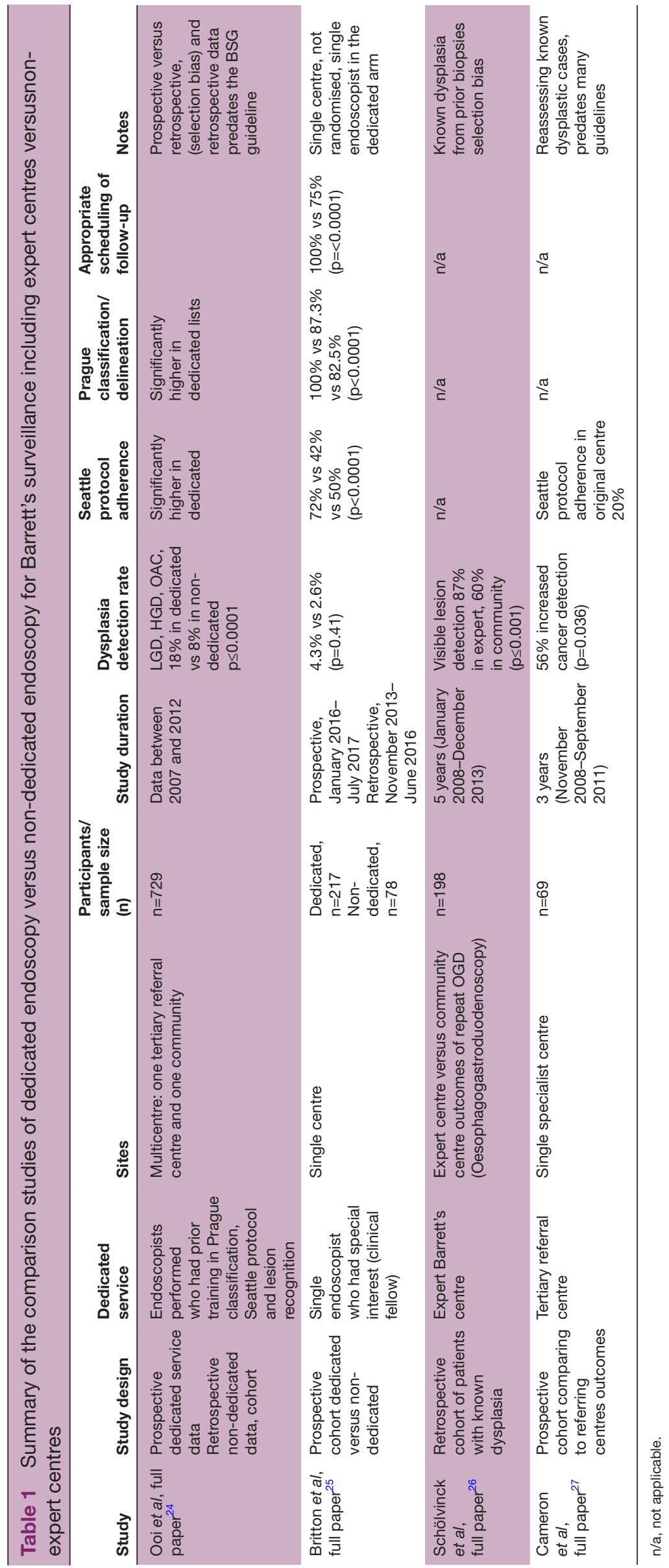


was identified which showed a significant difference in dysplasia detection rate. Ooi et al compared prospective data from two sites on a dedicated service, where four endoscopists who had prior training in the BSG guidelines and lesion detection performed surveillance at the two sites. ${ }^{24}$ This was then compared with retrospective data from a group of 47 different endoscopists from the same site over the prior 5 years. A total of 729 patients were reviewed; there was a significant difference in dysplasia detection rate (defined to include indefinite for dysplasia, low-grade dysplasia, high-grade dysplasia (HGD) and OAC) of $18 \%(26 / 142)$ in group A vs $8 \%$ $(45 / 587)$ in group B $(p<0.001)$. This study was extremely encouraging, showing markedly improved outcomes; however, there are some limitations; the BSG guideline bisected the retrospective data which could account for the lower figures, and there is a potential the prospective group benefited from this. No data were collected on inspection time, and it is possible the tertiary centre had selection bias with a saturated population with some cases referred in with known dysplasia.

Another cohort study by Britton et al compared a single-centre prospective cohort of patients undergoing Barrett's surveillance, either on a dedicated service $(\mathrm{n}=217)$ or a non-dedicated list $(\mathrm{n}=78) .{ }^{25}$ The dedicated service was defined as conducted by an endoscopist with a special interest in BO. This service was compared with those who attended lists performed by other operators, and with their retrospective data. Adherence to documentation guidelines was significantly better in the dedicated list with Prague classification reported (100\% vs $87.3 \%$ vs $82.5 \%, \mathrm{p}<0.0001)$ and location and number of biopsies $(99.5 \%$ vs $5.6 \%$ vs $6.9 \%, \mathrm{p}<0.0001)$. Adherence to the Seattle protocol was significantly greater in the dedicated list ( $72 \%$ vs $42 \%$ vs $50 \%, \mathrm{p}<0.0001)$, though this did not translate to significant differences in histology with intestinal metaplasia (IM) $(79.8 \%$ vs $73.1 \%, \mathrm{p}=0.12)$ and dysplasia/OAC $(4.3 \%$ vs $2.6 \%, \mathrm{p}=0.41)$. More patients were discharged from surveillance from the dedicated list due to comorbidity or performance status (13.4\%). This study was a single-centre cohort looking at a small number of patients, with a partly retrospective design, and no inspection time data were reported.

\section{Studies comparing expert centres with non-expert centres}

Rather than comparing a specific dedicated service within one trust to prior standard care, some studies have compared the outcomes for specialist or expert centres with community or non-expert endoscopy units. Schölvinck et $a l^{26}$ reviewed 198 patients who were referred from 37 community hospitals to a Dutch tertiary referral centre. Visible lesion detection was $87 \%$ in expert centres vs $60 \%$ in community cases. When HGD or OAC was found on a random sample, $76 \%$ had a lesion detected by the expert unit vs $50 \%$ when repeated in the community.

Cameron et $a l^{27}$ reviewed 69 cases who had been referred to a specialist $\mathrm{BO}$ centre for treatment of dysplasia. Adherence rates were poor in the community setting with 4/25 endoscopists adhering to Seattle protocol; $42 \%$ only noted any visible pathology on the referral endoscopy, which compared with $94 \%$ in the expert unit $(\mathrm{p} \leq 0.001)$. The expert unit confirmed all the prior known cancers and found an additional 10 cases at mapping. high-definition white-light endoscopy(HD-WLE) was used only in $57 \%$ of the community units and $14 \%$ used narrow band imaging (NBI), which might account for some of the missed lesions.

Limitations of these studies are that referrals to a specialist centre are a saturated population with known dysplasia, which introduces significant bias. Inspection time during endoscopy is an important factor influencing dysplasia detection, ${ }^{28}$ similar to colonoscopy adenoma detection; however, it is poorly reported in the aforementioned studies.

\section{Conference abstract data}

Given the paucity of published studies, conference abstract data are outlined in table 2 .

\section{WHAT ARE THE UNMET NEEDS OF PATIENTS WITH BO WHICH COULD BE ADDRESSED BY A DEDICATED SERVICE? Unmet need: clinical factors \\ Current surveillance practices: what improves outcomes?}

A systematic review and meta-analysis published in 2020 reviewed studies pertaining to adherence to $\mathrm{BO}$ guidelines. ${ }^{29}$ Fifty-six studies were included in qualitative analysis and 45 studies were used for quantitative synthesis/ meta-analysis. In this collection of international studies, they reviewed adherence to local guidance in a number of domains including total adherence, surveillance intervals, biopsy protocol and use of advanced imaging; landmark identification including length assessment using the Prague classification ${ }^{30}$; and histopathological results and correlated them with patient, endoscopist and endoscopy unit variables. Adherence ranged from $18 \%$ to $89 \%$ with the Seattle protocol. Factors associated with better adherence included university hospital endoscopy units, use of a dedicated list or service, shorter segment of $\mathrm{BO}$ and endoscopists who were employed as salaried clinicians opposed to those who were dependent on productivity. There was a wide variance in study designs; the studies were influenced by self-reporting of outcomes and country-based factors including different guidelines.

Adjuncts to support dysplasia detection and training the dedicated workforce

Currently, there is no specific assessment for competence in BO endoscopic surveillance beyond Joint Advisory Group accreditation in diagnostic UGI endoscopy. Though the quality Barrett's endotherapy guidelines and other guidelines for $\mathrm{BO}$ exist, ${ }^{31}{ }^{32}$ improving adherence to protocols as well as better recognition of visible lesions by a dedicated workforce could improve dysplasia detection.

\section{Training modules for $\mathrm{B} 0$ dysplasia detection}

The International Committee for Oesophagitis created a training module aimed at using videos to train 


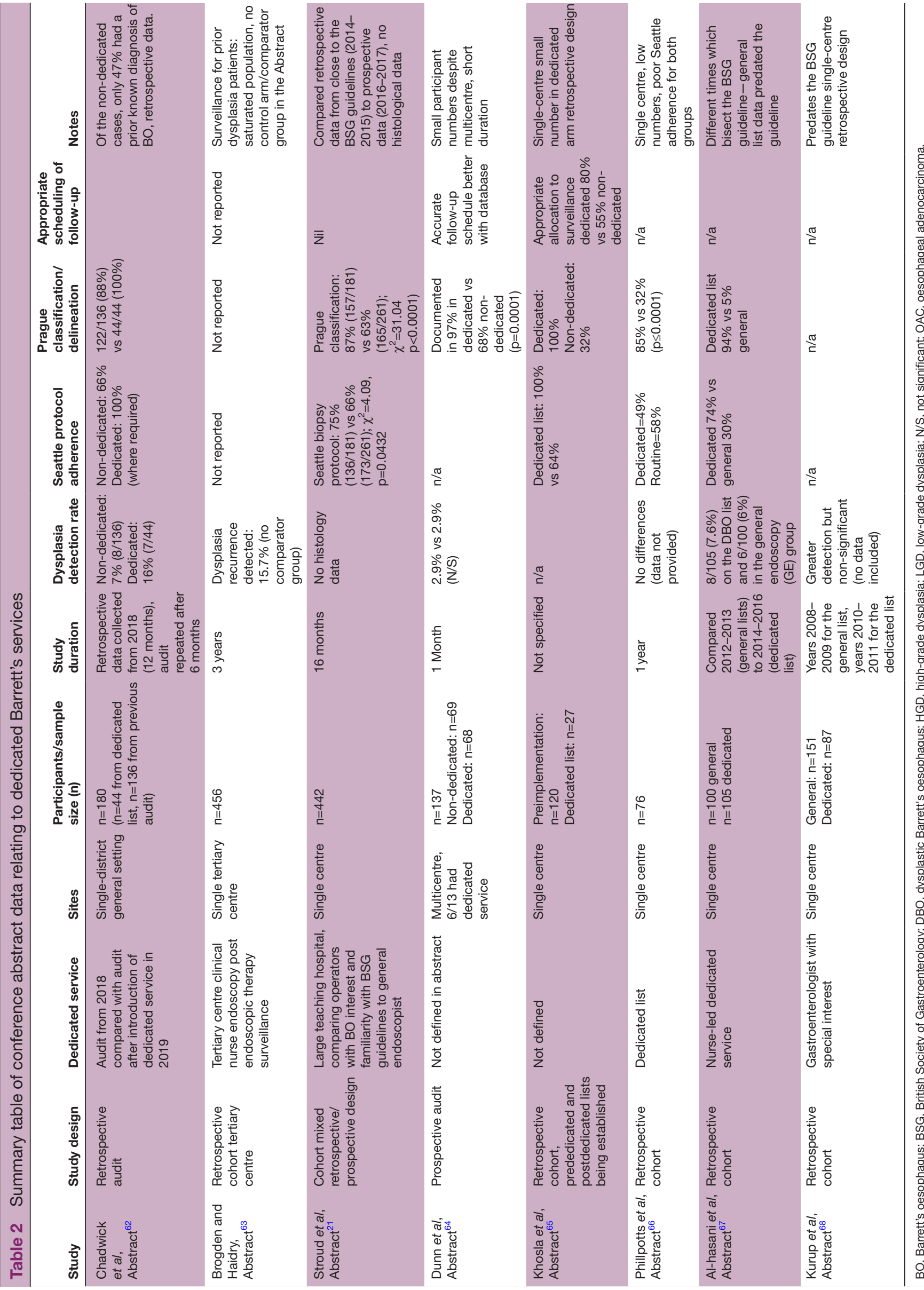


endoscopists to delineate Barrett's oesophagus-related neoplasm (BORN) ${ }^{33}$ Named the BORN training module, phase I involved collecting a series of endoscopy videos of $\mathrm{NDBO}$, and dysplastic $\mathrm{BO}$ which were annotated by three experts. They delineated any lesions and a 'sweet spot' for targeted biopsy. This formed a training series, comprising a test module and four training modules on which participants attempt to match the expert annotations, with mandated feedback between each module. Phase II evaluated BORN on an international cohort of 121 general endoscopists of varying experience. They showed marked sequential improvements between module 1 and module 4 with $46 \%$ in detection, $129 \%$ delineation and $106 \%$ relative delineation. The learning effect was independent of endoscopic expertise or country of origin.

\section{Advanced imaging techniques and acetic acid dye spray}

Advanced imaging techniques are a vital part of lesion recognition (eg, NBI, I-scan and volumetric laser endomicroscopy), enhancing abnormal vasculature and mucosal surface patterns, with many hoping targeted biopsies could replace Seattle protocol biopsies. ${ }^{34-36}$ Dilute acetic acid dye spray which causes a reversible reaction to the BO epithelium with early reversal in dysplastic tissues can be used to help detect dysplasia. It has been found to be cost effective if resulting in targeted samples alone in the surveillance population - particularly in higher risk groups. ${ }^{37}$ It is cheap and online learning has been shown that non-experts can learn how to use it with training programmes. ${ }^{38}$ Tholoor et al, using a per biopsy analysis, found a 14.7 -fold increase in neoplasia detection when endoscopists used ascetic acid-guided biopsies. ${ }^{39}$ An audit in Nottingham showed Seattle protocol still was important even with experts, ${ }^{40}$ but potentially a dedicated workforce, with adequate training in the use of adjuncts, could improve dysplasia detection.

\section{Artificial intelligence (Al)}

AI systems are being used (eg, I-scan and CADDIE) in $\mathrm{BO}$ to find dysplasia current methods miss. ${ }^{41-43}$ Lesion detection in $\mathrm{BO}$ is notoriously challenging for the uninitiated, and AI may provide some guidance; however, it requires a skilled workforce to use and interpret the signals from AI devices and to counsel patients about the potential limitations as responsibility remains with the operator. ${ }^{44}$ Machine learning tools could help to teach a dedicated workforce how to detect dysplasia. Seghal et al used I-scan, an endoscopy machine learning technology from Pentax on high-definition video of endoscopy procedures taken from patients undergoing surveillance. ${ }^{45}$ The experts reviewed the videos and scored them according to vascular pattern, visible lesions and whether they were non-dysplastic or dysplastic. Non-experts (GI trainees) and non-endoscopists (medical students) were then allowed to score and diagnose the videos, after which they watched a training video on how to detect dysplasia. Following this, they repeated their scoring of the videos. There was significantly improved sensitivity in the trainees' group (from $71 \%$ to $83 \%$ ) and specificity in the medical student group (from $31 \%$ to $49 \%$ ).

\section{Non-endoscopic dysplasia detection: Cytosponge}

The Cytosponge is a mesh sphere on a string which is swallowed in a gelatine capsule, and on withdrawal samples oesophageal mucosa from the length of the oesophagus, is safe and well tolerated by patients. ${ }^{46}$ Devised initially as a screening tool for primary care to detect the undiagnosed patients with $\mathrm{BO}$ in the community, the Cytosponge also provides an opportunity to support $\mathrm{BO}$ surveillance and reduce the patient burden, including after endotherapy. ${ }^{47}$ The Cytosponge-trefoil factor 3 versus usual care to identify $\mathrm{BO}$ in a primary care setting: a multicentre, pragmatic, randomised controlled trial (BEST3) ${ }^{48}$ comparing standard care to Cytosponge in the gastro-oesophageal reflux disease (GORD) population, found a 10 -fold increase in $\mathrm{BO}$ diagnosis, which raises the concern this may increase the burden of confirmatory endoscopy and endoscopic surveillance. However, work is under way to devise a biomarker panel to risk stratify patients, with the hope that low-risk groups could continue on Cytosponge surveillance alone or be appropriately discharged. ${ }^{49}$ Cytosponge is also now in use in the known BO surveillance population, which may allow streamlining of endoscopic assessment towards those at highest risk of dysplasia. ${ }^{50} \mathrm{~A}$ dedicated workforce is needed not only to assess endoscopically those found to have high-risk features but also to counsel patients about the different modalities and their limitations.

\section{Barriers to quality surveillance: clinician factors}

BO publications have often focused on clinical aspects, the performance of surveillance and technologies involved, yet clinician aspects have not been extensively explored. A survey ${ }^{13}$ of attitudes and practice of BO surveillance in UK gastroenterology clinicians was performed by the research group for the Aspirin and Esomeprazole in Barrett's Oesophagus (AspECT) trial. ${ }^{51}$ The research team sent surveys to 401 clinicians with 228 responses, of which 57 were from centres engaged in the AspECT study. They found $90 \%$ of responders would perform inadequate biopsy numbers; most would refer HGD to surgery; and $92 \%$ stated their lack of adherence to guidelines was due to the poor quality of evidence. In a follow-up survey, there was a significant increase in adherence to guidelines particularly in those engaged with the AspECT trial. Though this survey predated the most recent BSG guidelines, the authors suggest engagement with $\mathrm{BO}$ research improves practice, suggesting the important role of education for clinicians.

\section{Barriers to quality surveillance: departmental factors}

Inspection time when assessing $\mathrm{BO}$ segments improves dysplasia detection. ${ }^{28}$ There is a strong emphasis on withdrawal times in colonoscopy improving polyp detection, particularly in the bowel cancer screening scenario. ${ }^{52}$ The quality standards in UGI endoscopy suggest an inspection 
time of $1 \mathrm{~min} / \mathrm{cm}$ of $\mathrm{BO}$, yet $\mathrm{BO}$ surveillance can often be on varying endoscopy lists without adequate time allocation. ${ }^{32}$ Streamlining surveillance to those at greatest risk then scheduling them on dedicated lists with adequate time and a trained workforce (both the endoscopist and their team) may provide an environment more conducive to dysplasia detection. Streamlining is key, given the increasing demand on all aspects of endoscopy, which influences scheduling. ${ }^{53}$

\section{Cost implications of B0 surveillance}

Accurate use of the BSG guidelines implemented in 2014 has been shown to reduce the number of unnecessary surveillance procedures. At St Georges NHS trust, Hewett et al reviewed the BO population on their surveillance waiting lists, stratified them according to BO length according to the BSG guideline, and reviewed histology to check the presence of IM. ${ }^{54}$ They estimated accurately allocating surveillance would provide a saving over the subsequent 10 years of $£ 72033$ per annum, projecting across the NHS an approximate $£ 100$ million saved. An abstract from 2015 estimated the accurate scheduling of patients in York hospital would free 98 endoscopy slots with an annual cost saving of $£ 53503 .{ }^{55}$ Accurate BO scheduling has potentially dramatic cost-benefits, and benefits for patients by avoiding unnecessary invasive procedures; dedicated $\mathrm{BO}$ teams could oversee $\mathrm{BO}$ surveillance scheduling to facilitate these cost savings.

Beyond financial cost, endoscopy carries a huge environmental impact, from solid waste production, to water required for the processing of scopes, to processing costs of endoscopy samples. ${ }^{56} \mathrm{BO}$ contributes significantly to this with 2-5 yearly endoscopic surveillance, Seattle protocol biopsies, with the associated travel and departmental costs. No specific papers were found showing a link between dedicated services, streamlining surveillance and the environment, but it follows that avoiding unnecessary endoscopy and improving the quality of that which is performed may have benefits. Formal life-cycle analysis could be undertaken, exploring all aspects of the BO pathway to understand the full impact.

\section{Unmet need: patient factors \\ Dedicated BO clinics}

Only one study by Anagnostopoulos et al reviewed the outcomes of a $\mathrm{BO}$ clinic run by clinicians with an interest in $\mathrm{BO} .{ }^{58}$ Prospective data over 12 months were collected and questionnaires regarding patients understanding of BO were used. In the clinic, $17 \%$ had a treatment change, and $11 \%$ were discharged from surveillance. Their survey showed $22 \%$ of patients had had no information and $10 \%$ did not know they had BO with a similar figure believing they already had cancer. A key limitation was that it was an audit of a new intervention without a comparator. Likewise, the questionnaire was a single intervention and did not test for sustained improvements. However, they highlighted important deficits in patient knowledge and showed high discharge rates from inappropriate surveillance.

Britton et a $\tilde{p}^{9}$ carried out a quantitative study comparing the health-related quality of life of patients with $\mathrm{BO}$, both in non-dysplastic and dysplastic cases post-treatment, with healthy patients, those with GORD and patients with colonic polyps. Eligible cases totalled 687. Key findings were that those with NDBOs reported comparable levels of significant cancer worry (53\%) as those who had been treated for dysplastic $\mathrm{BO}$ and were clinically at greater risk $(50 \% \mathrm{p}=0.933)$. Patients who accurately perceived their cancer risk as low correlated with low levels of cancer worry, whereas anxiety, depression and cancer worry were significantly associated with worse control of GORD symptoms in patients with NDBO. This suggests that patients require greater support and education to reduce their cancer worry and empower them with knowledge of their actual cancer risk, and that good control of symptoms could help reduce worry and anxiety in this cohort.

A qualitative study in a single centre by the same group using semistructured interviews explored the experience of $\mathrm{BO}$ care for patients with NDBO. ${ }^{7}$ The interviews $(n=20)$ concentrated on follow-up needs, symptom burden both physical and psychological, and impact of the disease and surveillance. The results showed four themes:

- Burden of disease, including worry of cancer and endoscopy surveillance

- Follow-up care experiences.

- Follow-up needs.

- Disease-specific knowledge.

Most of the patients reported good symptom control; patients with a more central locus of control were able to manage their cancer worry better. Worry was relieved by good communication from their medical team, particularly early verbal reassurance about their surveillance and prompt biopsy results. When asked about follow-up, initial education and information were deemed inadequate and rushed. Disease-specific information overall was lacking, with most patients looking to other sources to self-educate; written information when provided was welcomed.

When asked about future considerations for follow-up care, patients valued seeing a specialist; most were openminded to nurse specialist care. They viewed a service run by a $\mathrm{BO}$ specialised team encompassing endoscopy and follow-up as more robust .

Other studies echo these findings: Murphy et al showed less than $50 \%$ of patients with BO-associated OAC knew about the diagnosis of $\mathrm{BO}$ despite multiple prior endoscopies. ${ }^{60}$ Stier $e t a l^{61}$ found patients with BO overestimated their risk of OAC with their lifetime risk perceived as $19 \%$. These studies suggest there is a burden of inadequate knowledge and cancer worry, dedicated $\mathrm{BO}$ follow-up services could provide education, risk counselling and offer a place for patient queries to be addressed beyond their initial diagnosis. 
Table 3 Outline of the unmet need in BO and the role dedicated services could take in addressing these issues

Unmet need for BO

\begin{tabular}{|c|c|c|}
\hline & Current issues & How dedicated BO services may address this need \\
\hline $\begin{array}{l}\text { Clinical factors } \\
\text { Dysplasia and } \\
\text { OAC detection. } \\
\text { Guideline } \\
\text { adherence. } \\
\text { Risk } \\
\text { stratification. }\end{array}$ & $\begin{array}{l}\text { Inadequate dysplasia detection, with } \\
\text { missed OAC up to } 12.7 \% \text { in UK data. } \\
\text { Outcomes for OAC remain poor and } \\
\text { rates are increasing in the UK. } \\
\text { Poor adherence to current guidelines } \\
\text { and biopsy surveillance protocols. } \\
\text { Service pressures on endoscopy, } \\
\text { making BO surveillance challenging to } \\
\text { achieve. } \\
\text { Widespread use of invasive surveillance } \\
\text { despite low overall risk of dysplasia and } \\
\text { OAC. }\end{array}$ & $\begin{array}{l}\text { Dedicated endoscopy lists run by clinicians with an interest in BO or } \\
\text { who have specific focused training, for example, BORN module. } \\
\text { Dedicated training for and use of adjuncts to support dysplasia } \\
\text { detection in BO, for example, artificial intelligence and acetic acid spray. } \\
\text { Adequate time for endoscopy procedures, for example, scheduling on } \\
\text { dedicated BO surveillance lists with adequate unit allocation. } \\
\text { Streamlining BO endoscopy to appropriate high-risk cases, including } \\
\text { the use of non-endoscopic sampling, for example, Cytosponge, and risk } \\
\text { stratification. May require dedicated team to review cases and counsel } \\
\text { patients. }\end{array}$ \\
\hline $\begin{array}{l}\text { Patient factors } \\
\text { Disease-specific } \\
\text { knowledge. } \\
\text { Worry of cancer. } \\
\text { Burden of } \\
\text { symptoms. } \\
\text { Burden of } \\
\text { surveillance. }\end{array}$ & $\begin{array}{l}\text { Poor understanding of their disease due } \\
\text { to incomplete education at diagnosis } \\
\text { and during the course of their condition. } \\
\text { Disproportionate cancer worry to } \\
\text { disease phenotype both for low-risk and } \\
\text { high-risk groups. } \\
\text { Lack of clear follow-up pathways/ways } \\
\text { to seek help during symptom flairs, } \\
\text { poor symptom control linked with higher } \\
\text { cancer worry. } \\
\text { Heavy burden of endoscopy, particularly } \\
\text { given the low risk of OAC in most cases. }\end{array}$ & $\begin{array}{l}\text { Dedicated BO clinics run by clinicians with an interest or specific } \\
\text { training in BO to address education needs, symptom needs, lifestyle } \\
\text { education and to discharge those who are inappropriate for surveillance, } \\
\text { for example, low risk or multicomorbid. } \\
\text { Dedicated BO follow-up in cases of need beyond the initial diagnosis } \\
\text { period, for example, via a helpline, email service or opt-in clinic/ } \\
\text { telephone clinic. } \\
\text { BO-specific patient-reported outcome measure to detect patients in } \\
\text { need of more education/support. } \\
\text { BO-specific education materials. } \\
\text { Augmentation of endoscopic surveillance with less invasive sampling } \\
\text { methods, for example, Cytosponge and risk stratification to reduce } \\
\text { endoscopy burden and streamline to high-risk groups; with education } \\
\text { and counselling by a dedicated, trained BO workforce. }\end{array}$ \\
\hline
\end{tabular}

BO, Barrett's oesophagus; BORN, Barrett's oesophagus-related neoplasm; OAC, oesophageal adenocarcinoma.

\section{SUMMARY}

OAC remains a significant cause of morbidity and mortality with high rates of preventable cases. Early diagnosis and treatment of BO-associated dysplasia and OAC offer an opportunity for improvement. Studies concentrate on interventions and dysplasia detection in the tertiary or specialist environment when more needs to be done to detect dysplasia in all units performing UGI endoscopy and BO surveillance. Early data from many published abstracts show encouraging improvements in adherence to surveillance guidelines with dedicated endoscopy services. However, more peer reviewed, multicentre prospective data looking at dysplasia and OAC detection are required, and there are currently no randomised controlled data. As well as technical factors, there are clinician-related factors which have not been well explored outside of surveys, and clinical endoscopists, trainees and surgeons have been missed from much of the data. Follow-up and education for patients with $\mathrm{BO}$ is lacking, and this results in a significant burden of cancer worry. Dedicated, patient-orientated services for $\mathrm{BO}$ are needed to address these issues (table 3). Tools to measure the effectiveness of interventions that are specific to this patient group should be developed to make sure advances in technological interventions are not to the detriment of the patient experience. An overhaul of current $\mathrm{BO}$ services is required to meet the needs of patients and improve clinical outcomes, with costs savings and reduced environmental burden possible if services are more efficient, and the correct patients are enrolled to surveillance with adequate education and support.
Twitter Elizabeth Ratcliffe @lil_ratcliffe

Acknowledgements The authors thank the members of the library team at Salford Royal Hospital, Northern Care Alliance Library Services for support with literature searching.

Contributors ER and YA devised the concept of the literature review. ER undertook the literature review and wrote the initial draft of the manuscript. All authors contributed to the review and revision of the final manuscript.

Funding The authors have not declared a specific grant for this research from any funding agency in the public, commercial or not-for-profit sectors.

Competing interests YA received research funding from Medtronic and Cancer Research UK; ER received research funding from Medtronic.

Patient consent for publication Not applicable.

Ethics approval This study does not involve human participants.

Provenance and peer review Not commissioned; externally peer reviewed.

Data availability statement Data sharing is not applicable as no datasets have been generated and/or analysed for this study. Not applicable.

Open access This is an open access article distributed in accordance with the Creative Commons Attribution Non Commercial (CC BY-NC 4.0) license, which permits others to distribute, remix, adapt, build upon this work non-commercially, and license their derivative works on different terms, provided the original work is properly cited, appropriate credit is given, any changes made indicated, and the use is non-commercial. See: http://creativecommons.org/licenses/by-nc/4.0/.

\section{ORCID iDs}

Elizabeth Ratcliffe http://orcid.org/0000-0001-6521-2133

John McLaughlin http://orcid.org/0000-0001-6158-5135

\section{REFERENCES}

1 Hvid-Jensen F, Pedersen L, Drewes AM, et al. Incidence of adenocarcinoma among patients with Barrett's esophagus. $N$ Engl J Med 2011;365:1375-83. 
2 Bhat S, Coleman HG, Yousef F, et al. Risk of malignant progression in Barrett's esophagus patients: results from a large populationbased study. J Natl Cancer Inst 2011;103:1049-57.

3 Uhlenhopp DJ, Then EO, Sunkara T, et al. Epidemiology of esophageal cancer: update in global trends, etiology and risk factors. Clin J Gastroenterol 2020;13:1010-21.

4 Cancer Research UK. Cancer research UK Oesophageal cancer statistics [Internet], 2020. Available: https://www.cancerresearchuk. org/health-professional/cancer-statistics/statistics-by-cancer-type/ oesophageal-cancer

5 Fitzgerald RC, di Pietro M, Ragunath K, et al. British Society of gastroenterology guidelines on the diagnosis and management of Barrett's oesophagus. Gut 2014;63:7-42.

6 Levine DS, Blount PL, Rudolph RE, et al. Safety of a systematic endoscopic biopsy protocol in patients with Barrett's esophagus. Am J Gastroenterol 2000;95:1152-7.

7 Britton J, Hamdy S, McLaughlin J, et al. Barrett's oesophagus: a qualitative study of patient burden, care delivery experience and follow-up needs. Health Expect 2019;22:21-33.

8 Britton J, Keld R, Prasad N, et al. Effect of diagnosis, surveillance, and treatment of Barrett's oesophagus on health-related quality of life. Lancet Gastroenterol Hepatol 2018;3:57-65.

9 Hinojosa-Lindsey M, Arney J, Heberlig S, et al. Patients' intuitive judgments about surveillance endoscopy in Barrett's esophagus: a review and application to models of decision-making. Dis Esophagus 2013;26:682-9.

10 Corley DA, Mehtani K, Quesenberry C, et al. Impact of endoscopic surveillance on mortality from Barrett's esophagus-associated esophageal adenocarcinomas. Gastroenterology 2013;145:312-9.

11 Ding YE, Li Y, He XK, et al. Impact of Barrett's esophagus surveillance on the prognosis of esophageal adenocarcinoma: a meta-analysis. J Dig Dis 2018;19:737-44.

12 Peters FP, Curvers WL, Rosmolen WD, et al. Surveillance history of endoscopically treated patients with early Barrett's neoplasia: nonadherence to the Seattle biopsy protocol leads to sampling error. Dis Esophagus 2008;21:475-9.

13 Das D, Ishaq S, Harrison R, et al. Management of Barrett's esophagus in the UK: overtreated and underbiopsied but improved by the introduction of a national randomized trial. $\mathrm{Am} \mathrm{J}$ Gastroenterol 2008;103:1079-89.

14 Abrams JA, Kapel RC, Lindberg GM, et al. Adherence to biopsy guidelines for Barrett's esophagus surveillance in the community setting in the United States. Clin Gastroenterol Hepatol 2009;7:736-42. quiz 710.

15 Visrodia K, Singh S, Krishnamoorthi R, et al. Magnitude of missed esophageal adenocarcinoma after Barrett's esophagus diagnosis: a systematic review and meta-analysis. Gastroenterology 2016;150:599-607.

16 van Putten M, Johnston BT, Murray LJ, et al. 'Missed' oesophageal adenocarcinoma and high-grade dysplasia in Barrett's oesophagus patients: A large population-based study. United Eur Gastroenterol $J$ 2018.

17 Crockett SD, Lipkus IM, Bright SD, et al. Overutilization of endoscopic surveillance in nondysplastic Barrett's esophagus: a multicenter study. Gastrointest Endosc 2012;75:23-31.

18 Royston C, Caygill C, Charlett A, et al. The evolution and outcome of surveillance of Barrett's oesophagus over four decades in a UK district general Hospital. Eur J Gastroenterol Hepatol 2016;28:1365-73.

19 Shaheen NJ, Falk GW, Iyer PG, et al. ACG clinical guideline: diagnosis and management of Barrett's esophagus. Am J Gastroenterol 2016;111:30-50.

20 Killcoyne S, Gregson E, Wedge DC, et al. Genomic copy number predicts esophageal cancer years before transformation. Nat Med 2020;26:1726-32.

21 Stroud S, Basu K. PTU-056 Generalist vs specialist: does dedicated endoscopy in barrett's oesophagus yield improved adherence to BSG guidance? Gut 2019;68:142.

22 Bird-Lieberman EL, Dunn JM, Coleman HG, et al. Population-based study reveals new risk-stratification biomarker panel for Barrett's esophagus. Gastroenterology 2012;143:927-35

23 Britton J, Gadeke L, Lovat L, et al. Research priority setting in Barrett's oesophagus and gastro-oesophageal reflux disease. Lancet Gastroenterol Hepatol 2017;2:824-31.

24 Ooi J, Wilson P, Walker G, et al. Dedicated Barrett's surveillance sessions managed by trained endoscopists improve dysplasia detection rate. Endoscopy 2017;49:C1.

25 Britton J, Chatten K, Riley T, et al. Dedicated service improves the accuracy of Barrett's oesophagus surveillance: a prospective comparative cohort study. Frontline Gastroenterol 2019;10:128-34.
26 Schölvinck DW, van der Meulen K, Bergman JJGHM, et al. Detection of lesions in dysplastic Barrett's esophagus by community and expert endoscopists. Endoscopy 2017;49:113-20.

27 Cameron GR, Jayasekera CS, Williams R, et al. Detection and staging of esophageal cancers within Barrett's esophagus is improved by assessment in specialized Barrett's units. Gastrointest Endosc 2014;80:971-83.

28 Gupta N, Gaddam S, Wani SB, et al. Longer inspection time is associated with increased detection of high-grade dysplasia and esophageal adenocarcinoma in Barrett's esophagus. Gastrointest Endosc 2012;76:531-8.

29 Roumans CAM, Der BRDV, Steyerberg EW, et al. Adherence to recommendations of Barrett' s esophagus surveillance guidelines : a systematic review and meta-analysis 2020:17-28.

30 Sharma P, Dent J, Armstrong D, et al. The development and validation of an endoscopic grading system for Barrett's esophagus: the Prague C \& M criteria. Gastroenterology 2006;131:1392-9.

31 Bennett C, Moayyedi P, Corley DA, et al. BOB CAT: a largescale review and delphi consensus for management of Barrett's esophagus with no dysplasia, indefinite for, or low-grade dysplasia. Am J Gastroenterol 2015;110:662-82.

32 Beg S, Ragunath K, Wyman A, et al. Quality standards in upper gastrointestinal endoscopy: a position statement of the British Society of gastroenterology (BSG) and association of upper gastrointestinal surgeons of great britain and ireland (AUGIS). Gut 2017;66:1886-1899.

33 JJGHM B, de Groof AJ, Pech O, et al. An Interactive Web-Based Educational Tool Improves Detection and Delineation of Barrett's Esophagus-Related Neoplasia. Gastroenterology 2019.

34 Verna C, Feyles E, Lorenzi L, et al. I-SCAN targeted versus random biopsies in Barrett's oesophagus. Dig Liver Dis 2014;46:131-4.

35 Trindade AJ, Raphael KL, Inamdar S, et al. Volumetric laser endomicroscopy features of dysplasia at the gastric cardia in Barrett's oesophagus: results from an observational cohort study. BMJ Open Gastroenterol 2019;6:e000340.

36 Everson MA, Lovat LB, Graham DG, et al. Virtual chromoendoscopy by using optical enhancement improves the detection of Barrett's esophagus-associated neoplasia. Gastrointest Endosc 2019;89:247-56

37 Bhandari P, Kandaswamy P, Cowlishaw D, et al. Acetic acidenhanced chromoendoscopy is more cost-effective than protocolguided biopsies in a high-risk Barrett's population. Dis Esophagus 2012;25:386-92.

38 Kandiah K, Chedgy FJQ, Subramaniam S, et al. International development and validation of a classification system for the identification of Barrett's neoplasia using acetic acid chromoendoscopy: the Portsmouth acetic acid classification (PREDICT). Gut 2018;67:2085-91.

39 Tholoor S, Bhattacharyya R, Tsagkournis O, et al. Acetic acid chromoendoscopy in Barrett's esophagus surveillance is superior to the standardized random biopsy protocol: results from a large cohort study (with video). Gastrointest Endosc 2014;80:417-24

40 Nachiappan A, Ragunath K, Card T, et al. Diagnosing dysplasia in Barrett's oesophagus still requires Seattle protocol biopsy in the era of modern video endoscopy: results from a tertiary centre Barrett's dysplasia database. Scand J Gastroenterol 2020;55:9-13.

41 Hussein M, González-Bueno Puyal J, Mountney P, et al. Role of artificial intelligence in the diagnosis of oesophageal neoplasia: 2020 an endoscopic odyssey. World J Gastroenterol 2020;26:5784-96.

42 Mori Y, Kudo S-E, Mohmed HEN, et al. Artificial intelligence and upper gastrointestinal endoscopy: current status and future perspective. Dig Endosc 2019;31:378-88.

43 Hussein M, Gonzalez-Bueno Puyal J, Brandao P, et al. Deep neural network for the detection of early neoplasia in Barrett 's oesophagus. Gastrointest Endosc 2020;91:AB250.

44 Hamade N, Sharma P. 'Artificial intelligence in Barrett's esophagus'. Ther Adv Gastrointest Endosc 2021;14:26317745211049964.

45 Sehgal V, Rosenfeld A, Graham DG, et al. Machine learning creates a simple endoscopic classification system that improves dysplasia detection in Barrett's oesophagus amongst non-expert endoscopists. Gastroenterol Res Pract 2018;2018:1872437.

46 Januszewicz W, Tan WK, Lehovsky K, et al. Safety and acceptability of esophageal Cytosponge cell collection device in a pooled analysis of data from individual patients. Clin Gastroenterol Hepatol 2019;17:647-56

47 Eluri S, Paterson A, Lauren BN, et al. Utility and cost-effectiveness of a Nonendoscopic approach to Barrett's esophagus surveillance after endoscopic therapy. Clin Gastroenterol Hepatol 2022;20:e51-63.

48 Fitzgerald RC, di Pietro M, O'Donovan M, et al. Cytospongetrefoil factor 3 versus usual care to identify Barrett's oesophagus 
in a primary care setting: a multicentre, pragmatic, randomised controlled trial. Lancet 2020;396:333-44.

49 Ross-Innes CS, Chettouh H, Achilleos A, et al. Risk stratification of Barrett's oesophagus using a non-endoscopic sampling method coupled with a biomarker panel: a cohort study. Lancet Gastroenterol Hepatol 2017;2:23-31.

50 et alHippisley-Cox J, Fitzgerald RC, Mei W. Project DELTA integrateD diagnostic solution for EarLy deTection of oesophageal cAncer Work Package 1 : Data Mining and Algorithm Development Research Protocol, 2021. Available: https://www.qresearch.org/ media/1284/ox39-delta-wp1-protocol_20210211.pdf

51 Jankowski JAZ, de Caestecker J, Love SB, et al. Esomeprazole and aspirin in Barrett's oesophagus (AspECT): a randomised factorial trial. Lancet 2018;392:400-8.

52 Lee TJW, Blanks RG, Rees CJ, et al. Longer mean colonoscopy withdrawal time is associated with increased adenoma detection: evidence from the bowel cancer screening programme in England. Endoscopy 2013;45:20-6.

53 Ravindran S, Bassett P, Shaw T, et al. National census of UK endoscopy services in 2019. Frontline Gastroenterol 2021;12:451-60.

54 Hewett R, Chan D, Kang J-Y, et al. New Barrett's oesophagus surveillance guidelines: significant cost savings over the next 10 years on implementation. Frontline Gastroenterol 2015;6:6-10.

55 Oka P, Kant P. PTU-323 Cost effective resourcing of a colitis chromoendoscopy service from implementing new bsg barrett's surveillance guidelines. Gut 2015;64:A203-4.

56 Gayam S. Environmental Impact of Endoscopy: "Scope" of the Problem. Am J Gastroenterol 2020;115:1931-2.

57 Gordon IO, Sherman JD, Leapman M, et al. Life cycle greenhouse gas emissions of gastrointestinal biopsies in a surgical pathology laboratory. Am J Clin Pathol 2021;156:540-9.

58 Anagnostopoulos GK, Pick B, Cunliffe R, et al. Barrett's esophagus specialist clinic: what difference can it make? Dis Esophagus 2006;19:84-7.
59 Britton J, Taxiarchi P, Martin G, et al. Comparative quantitative survey of patient experience in Barrett's oesophagus and other gastrointestinal disorders. BMJ Open Gastroenterol 2020;7:e000357.

60 Murphy SJ, Anderson LA, Johnston BT, et al. Patients with Barrett esophagus have poor understanding of the diagnosis and its implications. J Clin Gastroenterol 2008;42:431-2.

61 Stier MW, Lodhia N, Jacobs J, et al. Perceptions of risk and therapy among patients with Barrett's esophagus: a patient survey study. Dis Esophagus 2018;31. doi:10.1093/dote/dox109. [Epub ahead of print: 01 Apr 2018]

62 Chadwick G, Dyall L, Kyaw M. P20 Improving Barrett's surveillance in a DGH - dedicated lists are feasible and worthwhile. Gut 2021;70:A51-2.

63 May Brogden SE, Haidry R. O73 Introducing a clinical nurse endoscopist led Barrett's surveillance programme in a tertiary referral centre. Gut 2021;70:A40-1

64 Dunn JM, Wilson P, Chatu S, et al. PWE-082 Audit of Barrett's Oesophagus Surveillance in The London Cancer Alliance Structured Programmes with Dedicated Lists/Databases Improve Prague Scoring and Appropriate Follow Up Intervals: Abstract PWE082 Table 1. Gut 2016;65:A178.1.

65 Khosla S, Ratanshi N, Kelly M. Implementation of Dedicated Barrett's Oesophagus Endoscopic Diagnostic and Surveillance Lists in a District General Hospital. Int J Surg 2015;2020.

66 Phillpotts S, El MT, Preedy R. Diagnosis and documentation of findings bidirectional endoscopy with moderate sedation and carbon dioxide insufflation: a prospective observational study the efficacy for an additional intake of low volume polyethylene glycol (PEG) on the same day. Gastrointest Endosc 2016;83.

67 Al-hasani H, Sharp T, Ha SM LM, et al. A dedicated barrett's oesophagus endoscopy list improves the accuracy of endoscopic reporting and quality of biopsies - conference abstract. United Eur Gastroenterol J 2017;5.

68 Kurup AK, Mahmood R, Anderson M. PTU-025 Diagnostic Yield of a Dedicated Barrett'S Surveillance List using Trimodal Imaging: Abstract PTU-025 Table 1. Gut 2013;62:A52. 